\title{
IDENTIFICACIÓN BORROSA DE UN CULTIVO EXPERIMENTAL
}

\author{
M. Santos, B. Mendoza \\ Universidad Complutense de Madrid \\ msantos@ucm.es, bmendoz@gmail.com
}

\begin{abstract}
Resumen
En el campo de la producción agrícola es importante contar con un modelo del sistema que permita anticipar las diferentes situaciones debidas a la variabilidad de las condiciones climáticas y la incertidumbre de los datos que se manejan. Una técnica que permite tratar esta problemática es la lógica borrosa. En este trabajo, partiendo de datos registrados de un cultivo experimental de Maralfalfa en la Granja del Cabildo de Gran Canaria, se propone un modelo de reglas borrosas obtenido mediante el método de Wang-Mendel para estimar la producción de materia seca de este cultivo. Los resultados se han comparado con una regresión lineal. El modelo borroso obtenido se ajusta bien a los datos reales disponibles, lo que permitiría su uso para la predicción de la cosecha.
\end{abstract}

Palabras Clave: Agricultura de precisión, Lógica fuzzy, Identificación de Sistemas, Maralfalfa (Pennisetum Purpureum), Modelo borroso.

\section{INTRODUCCIÓN}

La producción forrajera debe de ser constante a lo largo de todo el año, para así poder tener alimento verde para el ganado y el excedente conservarlo para las épocas de un menor rendimiento del cultivo. Esto hace que sea necesario que el agricultor se pueda adelantar a diversas situaciones para que la cosecha sea la más ajustada posible a sus expectativas. Por ello, la aplicación de la agricultura de precisión junto con modelos que permitan predecir las variaciones en la producción son herramientas necesarias en la producción agraria actual.

La principal ventaja de la lógica borrosa reside en su capacidad para incorporar el conocimiento cualitativo que se tiene a priori sobre el comportamiento del sistema a tratar. Esto permite una representación cualitativa de sistemas complejos o con incertidumbre, para los que los modelos matemáticos resultan pobres e inadecuados. Los esquemas borrosos pueden usarse combinados con otras aproximaciones, tanto clásicas como inteligentes, o bien como metodologías únicas [16].
El presente trabajo se plantea obtener un modelo fácilmente interpretable, mediante el uso de la lógica borrosa, de la producción de materia seca de Maralfalfa (Pennisetum Purpureum). Para ello se han seleccionado diferentes variables de una muestra de datos reales, obtenidos de un cultivo experimental de esta planta en la Granja del Cabildo de Gran Canaria.

Para la obtención del modelo borroso se ha aplicado el método de Wang and Mendel (WM) [17] a un conjunto de variables explicativas relacionadas con el suelo, el clima y riego. El uso del modelo WM proporciona precisión en las predicciones y al mismo tiempo son modelos fáciles de entender por los no especialistas. En [4] se realiza una comparativa entre los diferentes métodos de obtención de modelos borrosos y el que se considera más apropiado desde el punto de vista de la interpretabilidad es el grupo entre los que se encuentra el método WM que, hasta donde hemos podido investigar, no ha sido aplicado anteriormente a este tipo de aplicación agrícola.

Se han obtenido buenos resultados y se han comparado con otros modelos analísticos. Con esto se pretende, una vez se tengan los suficientes datos y se haya probado más extensamente la validez del modelo, tener una ayuda para la toma de decisiones sobre el manejo de esta especie forrajera.

La estructura del artículo es la siguiente. En la s sección 2 se comentan brevemente algunos artículos relacionados. La información de partida se describe en la Sección 3. En la sección 4 se desarrolla el modelo borroso para la predicción de la Maralfalfa. Los resultados se exponen en la Sección 5. Las conclusiones y trabajos futuros terminan el trabajo.

\section{ESTADO DEL ARTE}

La predicción de cosechas es un tema que se ha abordado con diferentes técnicas por su utilidad dentro de lo que se denomina la agricultura de precisión, que permite mejorar los cultivos [7].

Aplicando técnicas estadísticas tradicionales, en [14] se realiza un estudio de la producción de materia seca de Maralfalfa usando como variables explicativas la frecuencia de corte y la dosificación de Nitrógeno. 
También las técnicas inteligentes, y más en concreto la lógica borrosa, se utilizan en diferentes campos de la agricultura de precisión como, por ejemplo, en la gestión de las cosechas $[6,12]$, modelos difusos del suelo y del agua de riego [3,11], la calidad de los alimentos, control de invernaderos, control de vehículos agrícolas, aplicación de herbicidas y abonos [1, 2, 15], etc. Una revisión del campo de aplicaciones del soft computing en agricultura puede encontrarse en [5].

Otros estudios relacionados con la predicción de la producción agrícola y las técnicas de la inteligencia artificial tratan de la producción de trigo en función del consumo de energía [6], realizado mediante el uso de técnicas neuroborrosas (ANFIS). Los autores de [10] estudiaron la producción de algodón mediante mapas cognitivos borrosos (FCM).

\section{MATERIALES}

La Maralfalfa (Pennisetum Purpureum $s p$ ) es un forraje de alto valor nutritivo, el cual se puede dar al ganado en verde o tras un proceso de ensilado o henificado [8]. Los requerimientos de este cultivo son muy elevados, por lo que es importante controlar todos los parámetros agroclimáticos si se quieren tener cosechas con una alta cantidad de materia seca.

Los datos obtenidos para este trabajo proceden de un estudio [9] llevado a cabo en una parcela de $336 \mathrm{~m}^{2}$ situada en la Granja Agrícola Experimental del Cabildo de Gran Canaria, en las coordenadas $\left(28^{\circ}\right.$ $\left.8^{\prime} 35.02^{\prime \prime N} ; 15^{\circ} 30^{\prime} 16.78^{\prime \prime O}\right)$. Se encuentra dividida en dos bloques con dos tratamientos de riego cada uno (T1 y T2), siendo el T1 el $100 \%$ de la ETP (evapotranspiración, pérdida de humedad de una superficie por evaporación directa junto con la pérdida de agua por transpiración de la vegetación) y el T2 una reducción del $40 \%$ de la ETP. Cada tratamiento consiste en cinco líneas de aproximadamente $21 \mathrm{~m}$ de longitud, coincidentes con las líneas de irrigación, con lo que el total es de 20 líneas en la parcela. Estas líneas están espaciadas $0.75 \mathrm{~m}$. En la parcela se ha instalado un sistema de riego enterrado con un caudal de $2.3 \mathrm{l} / \mathrm{h}$. Por otro lado, para monitorizar todos los parámetros se han instalado unos sensores de humedad y temperatura en el suelo, tanto superficiales como enterrados a $0.3 \mathrm{~m}$.

La toma de muestra del suelo se realiza en los primeros $0,2 \mathrm{~m}$ de profundidad en cada una de las veinte líneas, coincidiendo con los días de cosecha. Cada cosecha se realiza aproximadamente 56 días después de la anterior. Esta fecha dependerá de las condiciones agroclimáticas del momento; en primavera y verano se suele respetar, pero en otoño e invierno los cortes son más espaciados. La elección del momento de corte depende del valor nutritivo óptimo del cultivo.

Posteriormente, estas muestras son analizadas en el laboratorio para la determinación de los parámetros químicos más importantes. El carbón orgánico $(\mathrm{CO})$ y el Nitrógeno (N) se determinan mediante combustión seca con un analizador LECO CNS 2000. Las sales solubles se determinan por medido de la conductividad eléctrica EC1:5 (dS/m). El fósforo disponible ( $\mathrm{P} \mathrm{mg} / \mathrm{kg}$ ) se determina mediante la extracción del bicarbonato de sodio. Los cationes intercambiables (K, Ca, Mg y Na, meq 100 g-1; B, $\mathrm{Fe}, \mathrm{Cu}, \mathrm{Mn} \mathrm{y} \mathrm{Zn} \mathrm{mg} \mathrm{kg-1)} \mathrm{se} \mathrm{extraen} \mathrm{para} \mathrm{ser}$ analizados mediante Espectrofotómetro de emisión atómica (ICP-EOS).

\section{MODELO BORROSO}

De este conjunto de datos obtenidos se seleccionan tres tipos de variables que serán utilizadas para la elaboración del modelo borroso.

a) Variables representativas del suelo: se han seleccionado dos variables, Nitrógeno total y la Materia Orgánica. El Nitrógeno representa un factor importante de crecimiento y desarrollo para la planta. La Materia Orgánica es un indicador de la actividad biológica del suelo y es interesante además por las propiedades físicas y químicas que aporta al terreno.

b) Datos climatológicos: se usará la ETP (Evapotranspiración) como indicador de coeficiente de consumo hídrico.

La parcela experimental dispone de una estación meteorológica automática situada sobre el terreno para calcular la evapotranspiración PennmanMonteith (ETP, Water volumen evapotranspired, L. $\mathrm{m}^{-2}$ ), la integral termal $\left(\mathrm{TI},{ }^{\circ} \mathrm{C}\right.$ día), y la radiación $\left(\mathrm{rad}, \mathrm{MJ} \mathrm{m}^{-2}\right)$. En la fórmula 1 se expresa la ecuación de Pennman-Monteith para el cálculo de la ETP:

Donde:

$$
\lambda E T_{0}=\frac{\Delta\left(R_{n}-G\right)+\rho c_{p}\left(e_{a}-e_{d}\right) / r_{a}}{\Delta+\gamma\left(1+\frac{r_{c}}{r_{a}}\right)}
$$

- $\lambda E T_{0}:$ Flujo del calor latente de evaporación $\left[\mathrm{kJ} \cdot \mathrm{m}^{2} \cdot \mathrm{s}^{-1}\right]$

- Re: Flujo de la radiación neta en superficie $\left[\mathrm{kJ} \cdot \mathrm{m}^{2} \cdot \mathrm{s}^{-1}\right]$

- G: Flujo térmico del suelo $\left[\mathrm{kJ} \cdot \mathrm{m}^{2} \cdot \mathrm{s}^{-1}\right]$

- $\rho$ : Densidad atmosférica $\left[\mathrm{kg} \cdot \mathrm{m}^{3}\right]$

- $\mathrm{c}_{\mathrm{p}}$ : Calor específico del aire húmedo $\left[\mathrm{kJ} \cdot \mathrm{kg}^{-1}{ }^{\circ} \mathrm{C}^{-1}\right]$

- $\left(\mathrm{e}_{\mathrm{a}}-\mathrm{e}_{\mathrm{d}}\right)$ : Déficit de presión de vapor [kPa]

- $\mathrm{r}_{\mathrm{c}}$ : resistencia de la cubierta vegetal $\left[\mathrm{s}^{\mathrm{m}} \mathrm{m}^{-1}\right]$ 
- $\Delta$ : Pendiente de la curva de presión de vapor $[\mathrm{kPa}$ $\left.{ }^{\circ} \mathrm{C}^{-1}\right]$

- $\gamma$ : Constante psicrométrica $\left[\mathrm{kPa}{ }^{\circ} \mathrm{C}^{-1}\right]$

- $\lambda$ : Calor latente de vaporización $\left[\mathrm{MJ}^{\mathrm{k}} \mathrm{kg}^{-1}\right]$

c) Sistema de irrigación: el riego se aplica mediante un sistema de goteo enterrado (subsurface drip irrigation: SDI) con goteros integrados autocompensantes (rango 1,5 a 4,0 bar), antidrenantes y con mecanismos anti-sifón, separados a $0.5 \mathrm{~m}$ y con un caudal nominal de $2.3 \mathrm{~L} / \mathrm{h}$. El riego se produce dos veces al día durante 20 minutos (T1) y 12 minutos (T2). Cada tratamiento tiene su medidor de flujo con lecturas semanales.

Como variable de salida se tiene la producción de materia seca (MS) por metro cuadrado. $\mathrm{La}$ determinación de MS se realiza en estufa a $60^{\circ} \mathrm{C}$ hasta llegar a peso constante.

Como se ha indicado, para el modelo se usará como variable dependiente la producción de materia seca (Prod) y las restantes variables (materia orgánica Materia-, Nitrógeno total -Nitro-, ETP acumulado ETP- y consumo hídrico - Riego-) serán las entradas (Figura 1).

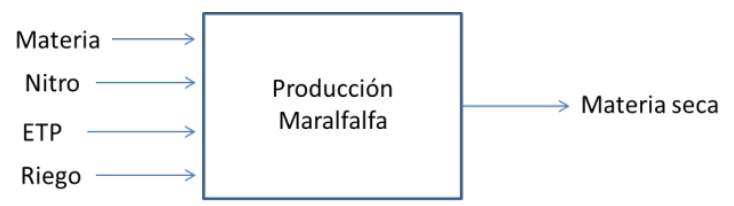

Figura 1: Diagrama de bloques del modelo

De un análisis de las variables se puede saber que existe una correlación entre las variables Materia y Nitro, y entre ETP y Riego.

\subsection{MODELO BORROSO DE WANG- MENDEL}

De entre los diferentes métodos de identificación borrosa se usará el de Wang-Mendel (WM) [17], siendo el modelo resultante de tipo Mamdani.

Para el caso de dos entradas y una salida, suponiendo que se realizan $\mathrm{n}$ experimentos, se obtiene un conjunto de datos de entrada-salida $\left(x_{1}^{(1)}, x_{2}^{(1)} ; y^{1}\right),\left(x_{1}^{(2)}, x_{2}^{(2)} ; y^{2}\right), \ldots\left(x_{1}^{(n)}, x_{2}^{(n)} ; y^{n}\right)$, donde $\operatorname{los} x_{1}, x_{2}$ son las entradas e $\boldsymbol{y}$ son las salidas y el superíndice es el número del experimento, desde 1 hasta $\mathrm{n}$. La tarea es generar un conjunto de reglas a partir de estos datos y usar estas reglas para determinar un mapeado: $f:\left(x_{1}, x_{2}\right) \rightarrow y$

El método consiste en cinco etapas para generar las reglas a partir de los datos numéricos. Para aplicar el método WM a la identificación del modelo objeto del trabajo se ha utilizado el paquete frbs [13] del entorno de programación estadístico $\mathrm{R}$.

La configuración del FIS (Fuzzy Inference System) usado en la identificación del sistema es la siguiente:

- Funciones de pertenencia Gaussianas,

- Defuzzificación por método de la media ponderada (WAM)

- t-norma: Min

- s-Norma: Max

- Tipo de implicación: Zadeh.

Las cinco etapas del método se describen y aplicación a continuación.

\subsubsection{Paso 1: Dividir el espacio de entradas- salidas en regiones borrosas.}

Asumiendo que los intervalos del dominio de las variables de entrada $\boldsymbol{x}_{\boldsymbol{i}}$ y la de salida $\mathbf{y}$ son $\left[x_{i}^{-}, x_{i}^{+}\right]$e $\left[y^{-}, y^{+}\right]$, se divide el intervalo en $\mathrm{N}$ regiones $(\mathrm{N}$ no necesariamente igual para todas las variables), asignando a cada región una función de pertenencia.

En la tabla 1 se muestran los rangos de las variables de entrada y salida del modelo.

Tabla 1: Rango de las variables del modelo.

\begin{tabular}{|l|lllll|}
\hline & Materia & Nitro & ETP & Riego & Prod \\
\hline $\min$ & 2,18 & 0,16 & 149,04 & 0,07 & 0,26 \\
\hline $\max$ & 3,71 & 0,28 & 373,08 & 0,31 & 1,23 \\
\hline
\end{tabular}

Una vez normalizados los datos, se divide el espacio en tres intervalos (small, medium y large) tanto para las variables de entrada como para la de salida. Es decir, el número de conjuntos borrosos por variables es $\mathrm{N}=3$. Con lo que la división del espacio de entrada-salida es (figura 2):
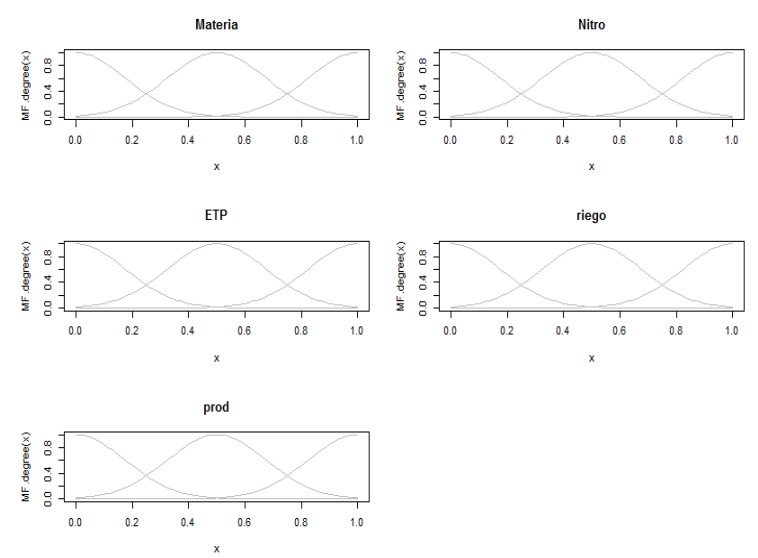

Figura 2: Partición del espacio de entradas (materia, Nitro, ETP y riego) y salida (prod), respectivamente 


\subsubsection{Paso 2: Generar las reglas borrosas a partir de los pares de datos}

Inicialmente se determinan los grados de pertenencia de $x_{1}^{(i)}, \ldots, x_{n}^{(i)} ; y^{i}$, siendo $\mathrm{n}=4$ el número de variables de entrada del modelo e $i$ cada uno de los experimentos (cortes $=9$ ), dando un total de 36. A continuación se asignan esos datos a la región con mayor grado de pertenencia, llamado $\mathrm{m}_{\mathrm{A}}\left(\mathrm{x}_{1}\right)$ para la variable $\mathrm{x}_{1}$ en la región $\mathrm{A}$. Por último se obtiene una regla para cada par de entrada-salida.

Las reglas generadas de esta forma son del tipo,

$$
\text { IF } \mathrm{x}_{1} \text { es } \mathrm{A} \text { And } \mathrm{x}_{2} \text { es } \mathrm{B} \text { THEN y es } \mathrm{C}
$$

En este sistema se tienen cuatro antecedentes y un consecuente. Aunque inicialmente podrían generarse 36 reglas, al aplicar los pasos del algoritmo algunas de ellas se pueden combinar para reducir la dimensión de la tabla de reglas.

\subsubsection{Paso 3: Asignar a cada regla un grado}

Dado que existe un determinado número de pares de datos de entrada-salida, y que para cada par se genera una regla, es probable que existan reglas en conflicto (por ejemplo con los mismos antecedentes y distintos consecuentes). Una manera de resolver estos conflictos es asignar un grado a cada regla generada $\mathrm{y}$, en caso de reglas en conflicto, aceptar la de mayor grado. De esta manera se resuelve el conflicto y también se reduce el número de reglas.

El método WM usa la estrategia del producto para asignar el grado a cada regla. Para el caso de dos variables y una salida, el grado (D) asignado a cada regla se define como:

$$
\mathrm{D}(\text { regla })=\mathrm{m}_{\mathrm{A}}\left(\mathrm{x}_{1}\right) * \mathrm{~m}_{\mathrm{B}}\left(\mathrm{x}_{2}\right) * \mathrm{~m}_{\mathrm{C}}(\mathrm{y})
$$

\subsubsection{Paso 4: Crear la base de reglas borrosas combinada}

Para el caso bidimensional, la base de reglas es una matriz donde las filas corresponden a una variable $\left(\mathrm{x}_{2}\right)$ y las columnas a otra $\left(\mathrm{x}_{1}\right)$. Para rellenar cada celda se sigue la siguiente estrategia: se asignan las reglas a la base y si hay una celda con más de una regla, se usa la regla que posea mayor grado.

En nuestro caso, una vez eliminados los conflictos, se obtienen 27 reglas con los correspondientes grados. Además los grados D de las reglas 26 y 27 son cero por lo que se eliminan de la base de reglas (con bajo riego y bajo nivel de nitrógeno la producción es baja en todos los casos), quedando ésta como se muestra en la Tabla 2.
Tabla 2: Grado (D) de cada una de las reglas.

\begin{tabular}{|ll|ll|}
\hline REGLA & GRADO D & REGLA & GRADO D \\
\hline 1 & 0,777 & 15 & 0,412 \\
\hline 2 & 0,775 & 16 & 0,377 \\
\hline 3 & 0,635 & 17 & 0,359 \\
\hline 4 & 0,635 & 18 & 0,323 \\
\hline 5 & 0,635 & 19 & 0,306 \\
\hline 6 & 0,635 & 20 & 0,221 \\
\hline 7 & 0,632 & 21 & 0,221 \\
\hline 8 & 0,595 & 22 & 0,189 \\
\hline 9 & 0,500 & 23 & 0,131 \\
\hline 10 & 0,500 & 24 & 0,082 \\
\hline 11 & 0,483 & 25 & 0,021 \\
\hline 12 & 0,448 & 26 & 0,000 \\
\hline 13 & 0,430 & 27 & 0,000 \\
\hline 14 & 0,430 & & \\
\hline
\end{tabular}

\subsubsection{Paso 5: Aplicar el sistema de inferencia borroso}

Este paso consiste en aplicar el sistema de inferencia borroso tipo Mamdani para obtener las salidas a partir de las reglas.

Para determinar la salida, y dadas las entradas $\left(\mathrm{x}_{1}, .\right.$. , $\mathrm{x}_{\mathrm{n}}$ ), primero se combinan los antecedentes de la $i$ ésima regla borrosa usando la operación producto para determinar el grado $m_{O i}^{i}$ de la salida correspondiente:

$$
m_{O^{i}}^{i}=m_{I_{1}^{i}}\left(x_{1}\right) * . . * m_{I_{n}^{i}}\left(x_{n}\right)
$$

Donde $\mathrm{O}^{\mathrm{i}}$ denota la región de salida de la regla $i$, e $I_{j}^{i}$ es la región de entrada de la regla $i$ para la variable $j$.

Siguiendo el ejemplo de dos variables de entrada tendríamos para la regla 1 :

$$
\mathrm{m}_{\mathrm{C}}{ }^{1}=\mathrm{m}_{\mathrm{A} 1}\left(\mathrm{x}_{1}\right) * \mathrm{~m}_{\mathrm{B} 1}\left(\mathrm{x}_{2}\right)
$$

Posteriormente se usa la fórmula de defuzzificación por el método del centroide para determinar la salida:

$$
y=\frac{\sum_{i=1}^{K} m_{O^{i}}^{i} \bar{y}^{i}}{\sum_{i=1}^{K} m_{O^{i}}^{i}}
$$

Donde $\bar{y}^{i}$ es el valor centro de la región $\mathrm{O}^{\mathrm{i}}$ (definido como el punto de menor valor absoluto entre los puntos cuyo valor de pertenencia a la función es igual a 1), y $\mathrm{K}$ es el número de regla borrosa. 
Para cada una de las 36 observaciones se obtiene un valor estimado de la producción de materia seca. El error, dados por los valores de los residuos, es muy bajo y llega a ser cero en alguno de los casos.

Para estimar la variabilidad de los modelos obtenidos se puede realizar el ajuste repetidamente con diferentes muestras. La validación cruzada se usa para estimar el error asociado con cada método durante el aprendizaje, y así evaluar el modelo. En este caso el valor de la estimación es el siguiente, siendo $\mathrm{n}$ el número de muestras.

$$
C V=\frac{1}{n} \sum_{i=1}^{n} \text { Error }
$$

Dado el pequeño número de registro para la identificación del que se dispone, la validación se hará usando el método LOO-CV para chequear el rendimiento tanto del modelo borroso como de la regresión lineal usada para comparar, tomando como medida del rendimiento la raíz del error cuadrático medio (RMSE).

Según los datos disponibles, y aplicando la metodología anteriormente descrita, la recta de regresión final es:

$$
\begin{gathered}
\text { Prod }=0,437-0,142 \text { Materia }+ \\
2,572 \text { Nitro }-0,001 \text { ETP }+1,949 \text { riego }
\end{gathered}
$$

Los resultados de producción (prod) obtenidos para cada una de los conjuntos de variables de entrada (Materia, Nitro, ETP y riego) para el modelo borroso WM, para la regresión lineal calculada y las mediciones reales en cada uno de los 36 cortes se muestran en la Figura 3.

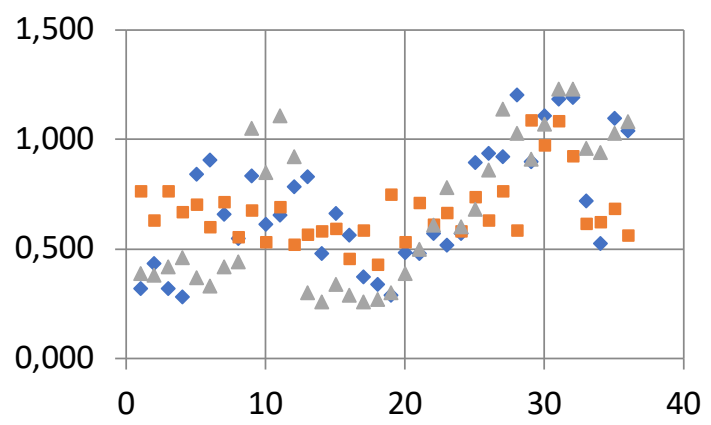

Figura 3: Comparativa de resultados de la producción $\left(\mathrm{Kg} / \mathrm{m}^{2} \mathrm{MS}\right)$ para el modelo borroso (azul), la regresión lineal (rojo) y la producción medida real (verde) para cada una de las 36 muestras (cortes)

En la gráfica se observa que el modelo WM sigue con más fidelidad las tendencias de la producción real que la recta de regresión.
El error RMSE, para la cross-validation y el modelo borroso y la regresión son los mostrados en la tabla 3 .

Tabla 3: RMSE del modelo borroso y la regresión.

\begin{tabular}{|l|ll|}
\hline & Modelo Borroso & Regresión Lineal \\
\hline RMSE LOO & 0,33 & 0,34 \\
\hline RMSE Modelo & 0,23 & 0,29 \\
\hline
\end{tabular}

Como se observa, en ambos casos es mejor el resultado obtenido por el modelo borroso, tanto para el modelo final como durante el entrenamiento, lo que indica que para nuevos datos el resultado del modelo borroso será mejor que la regresión.

\section{CONCLUSIONES Y TRABAJOS FUTUROS}

En el trabajo se muestra una metodología para la identificación mediante lógica borrosa de la producción de materia seca de Maralfalfa, teniendo en cuenta un pequeño número de variables.

A partir de datos reales, se ha obtenido un modelo descrito por un conjunto de reglas fácilmente interpretables por los expertos y con resultados de predicción mejores que los de una regresión lineal. Estos modelos pueden irse actualizando tanto con la aportación de conocimiento por parte de expertos como con nuevos datos.

El modelo se basa en la aplicación del método de Wang-Mendel, y se ha diseñado para cuatro variables de entrada, obteniendo como salida la producción de materia seca de Maralfalfa, que es la componente que se quería modelar del cultivo.

Como trabajo futuro, se propone realizar un análisis modificando el número de variables del modelo, para discernir cuáles son las más relevantes para una mejor predicción de la cosecha.

Dado que el número de muestras no es suficientemente grande como para obtener conclusiones definitivas, sería deseable obtener un número mayor de registros, incorporar nuevas variables ordenándolas en función de su importancia, extrapolar las reglas sobre regiones no cubiertas por los datos, o ajustar los parámetros del modelo predictivo.

\section{English summary}

FUZZY IDENTIFICATION OF AN
EXPERIMENTAL CROP 


\begin{abstract}
In the agricultural field it is important to have a model of the crop production in order to forecast possible situations due to the variability of the weather conditions and the uncertainty in the measures. One technique that allows us to deal with this problem is fuzzy logic. In this work, based on real data obtained from an experimental crop of Elephant Grass in the Gran Canaria Cabildo Farm, a fuzzy model is obtained by the Wang-Mendel method to estimate the dry matter production of this crop. The results have been compared with a linear regression. The fuzzy model is well adjusted to the available real data, thus it can be used for the prediction of the harvest.
\end{abstract}

Keywords: Precision Agriculture, Fuzzy Logic, Systems Identification, Elephant Grass (Pennisetum Purpureum), Fuzzy Model.

\section{Referencias}

[1] da Silva, A. A., Silva, I. A., Teixeira Filho, M., Buzetti, S., Teixeira, M. (2014) "Estimativa da produtividade de trigo em função da adubação nitrogenada utilizando modelagem neuro fuzzy". Revista Brasileira de Engenharia Agrícola e Ambiental, 180-187.

[2] Burgos-Artizzu, X. P., Ribeiro, A., Santos. M., (2007) "Controlador Borroso Multivariable para el Ajuste de Tratamientos en Agricultura de Precisión", Revista Iberoamericana de Automática e Informática Industrial 4(2), 6471.

[3] Khan, F., Shabbir, F., Tahir, Z. (2014) “A fuzzy approach for water security in irrigation system using wireless sensor network", Science International, 26(3), pp. 1065.

[4] Guillaume, S. (2002) "Designing fuzzy inference systems from data: An interpretability-oriented review". IEEE Transactions on Fuzzy Systems 9(3), pp. 426443.

[5] Huang, Y., Lan, Thomson, S.J., Fang, A., Hoffmann, W.C., Lacey, R.E. (2010) "Development of soft computing and applications in agricultural and biological engineering", Computers an Electronics in Agriculture 71(2), pp. 107-127.

[6] Khoshnevisan, B., Rafiee, S., Omid M., Mousazadeh, H. (2014) "Development of an intelligent system based on ANFIS for predicting wheat grain yield on the basis of energy inputs", Information Processing in Agriculture 1(1), pp. 14-22.
[7] Macedo-Cruz, A., Pajares, G., Santos, M., Villegas-Romero, I. (2011) "Digital Image Sensor-Based Assessment of the Status of Oat (Avena sativa L.) Crops after Frost Damage", Sensors 11(6), pp. 6015-6036.

[8] Márquez, F., Sánchez, J., Urbano, D., Dávila, C. (2007) "Evaluación de la frecuencia de corte y tipos de fertilización sobre tres genotipos de pasto elefante (Pennisetum purpureum) 1. Rendimiento y contenido de proteína", Zootecnia tropical, 25(4), pp. 253-259.

[9] Mendoza-Grimón, V., Hernández-Moreno, J.M., Palacios-Díaz, M.D.P. (2015) "Improving Water Use in Fodder Production". Water 7(6) pp. 2612-2621.

[10] Papageorgioua, A.T. Markinos G. (2011) "Fuzzy cognitive map based approach for predicting yield in cotton crop production as a basis for decision support system in precision agriculture application", Applied Soft Computing 11(4), pp 3643-3657.

[11] Patil, P. and Desai, B.L. (2013) "Intelligent irrigation control system by employing wireless sensor networks", International Journal of Computer Applications, 79(11) pp. 33-40.

[12] Pydipati R., Burks, T.F., Lee, W.S. (2005) "Statistical and neural network classifiers for citrus disease detection using machine vision", Transactions of the ASAE, 48(5) pp. 2007-2014.

[13] Riza. L.S, Bergmeir, C., Herrera, F., Benítez, J.M. (2015) frbs: Fuzzy rule-based systems for classification and regression in R. Journal of Statistical Software, 65(6).

[14] Larduet, R., Ramos, N. (2013) "Modelling of dry matter yield of Pennisetum purpureum cv. king grass with different cutting frequencies and N fertilizer dosages", Cuban Journal of Agricultural Science, 47(3).

[15] López, M. D. R., Uribe, J. D. S., Vásquez, L. M. L. (2015) "Una estrategia de innovación en fertilizantes orgánicos mediante lógica difusa”, Revista Facultad Nacional de Agronomia Medellin, 68(1), 7423.

[16] Santos, M. (2011) "Un enfoque aplicado del control inteligente", Revista Iberoamericana de Automática e Informática Industrial, 8, pp. 283296.

[17] Wang, L. X., \& Mendel, J. M. (1992) "Generating fuzzy rules by learning from examples", IEEE Transactions on Systems, Man, and Cybernetics, 22(6), pp. 1414-1427.

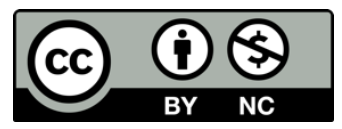

(C) 2018 by the authors. Submitted for possible open access publication under the terms and conditions of the Creative Commons Attribution CC-BY-NC 3.0 license (https://creativecommons.org/licenses/by-nc/3.0). 